\title{
Acknowledgment of Reviewers, 1996
}

The success of Phytopathology depends on the quality of manuscripts submitted by authors and on the care and competence with which they are reviewed. It is the policy of the Editorial Board to solicit reviews for manuscripts from specialists who are most qualified to review them. In addition to members of the Editorial Board, the individuals listed below provided constructive critical reviews of one or more manuscripts during the past year. Their names are published here in grateful appreciation of their contribution to the journal and to the science of phytopathology. Publication no. P-1997-1125-010

Abramson, D., Agriculture and Agr-Food Canada, Winnipeg, MB, Canada

Aist, J. R., Cornell University, Ithaca, NY

Aldwinckle, H., Cornell University, Geneva, NY

Jim Anderson, University of Toronto, Mississauga, ON, Canada

Andrews, J., University of Wisconsin, Madison, WI

Appel, D., Texas A\&M University, College Station,TX

Aylor, D., Conn. Agric. Exp. Station, New Haven, CT

Backman, P., Auburn University, Auburn, AL

Bailey, B., USDA, ARS, Beltsville, MD

Baker, C. J., USDA, ARS, Beltsville, MD

Banuett, F., School of Medicine, San Francisco, CA

Beaulieu, C., University of Sherbrooke, QC, Canada

Beer, S., Cornell University, Ithaca, NY

Belanger, R., Universite Laval, QC, Canada

Benhamou, N., Universite Laval, QC, Canada

Berbee, M., University of British Columbia, Vancouver, BC, Canada

Bergmann, C., University of Georgia, Athens, GA

Biggs, A. W. Virginia University, Kearneysville, WV

Bisaro, D. M., Ohio State University, Columbus, $\mathrm{OH}$

Blanchette, R., University of Minnesota, St. Paul, MN

Boland, G., University of Guelph, Guelph, ON, Canada

Bonman, J. M., DuPont KK, Tsukuba, Ibaraki, Japan

Boudreau, M., Warren Wilson College, Asheville, NC

Bowers, J. H., USDA, ARS, Beltsville, Beltsville, MD

Bowers, J., University of Minnesota, St. Paul, MN

Braun, E., Iowa State University, Ames, IA

Brlansky, R., University of Florida, Lake Alfred, FL

Brodie, W., Cornell University, Ithaca, NY

Broglie, R., E. I. DuPont de Nemours \& Co, Wilmington, DE

Brown, G., University of California, Davis, CA

Brown, J., John Innes Centre for Plant Science Research,

Norwich, England

Brown, J. K., University of Arizona, Tucson, AZ

Brown, R.L., USDA, ARS, Southern Regional Research Center,

New Orleans, LA

Brlansky R., University of Florida, Lake Alfred, FL

Bruckart, W., USDA-FDWSRU, Frederick, MD

Bushnell, W., University of Minnesota, St. Paul, MN

Campbell, C. L., North Carolina State University, Raleigh, NC

Candresse, T., INRA Station de Pathologie Vegetale, France

Carling, D., University of Alaska, Palmer, AK

Carris, L., Washington State University, Pullman, WA

Carson, M., North Carolina State University, Raleigh, NC

Chase T. E., University of South Dakota, Brookings, SD

Chatterjee, A., University of Missouri, Columbia, MO

Chellemi, D., University of Florida, Quincy, FL

Chen, W., Illinois Natural History Survey, Champaign, IL

Chen, X., Washington State University, Pullman, WA

Chong, J., Agriculture and Agri-Food-Canada, Winnipeg, MB, Canada

Ciufetti, L., Oregon State University, Corvallis, OR

Clayton, M., University of Wisconsin, Madison, WI
Correll, J. C., University of Arkansas, Fayetteville, AR

Cotty, P. J., USDA, ARS, Southern Regional Research Center, New Orleans, LA

Cubeta, M., North Carolina State University, Plymouth, NC

Cunfer, B., Georgia Experiment Station, Griffin, GA

Cuppels, D. A. Agriculture and Agri-Food Canada, London, ON, Canada

De Zoeten, G. A., Michigan State University, East Lansing, MI

Dickman, M., University of Nebraska, Lincoln, NE

Dion, P., University of Laval, Quebec, Canada

Dolja, V., Oregon State University, Corvallis, OR

Domier, L., University of Illinois, Urbana, IL

Dunkle, L., Purdue University, West Lafayette, IN

Duvick, J., Pioneer Hi-Bred International, Johnston, IA

Ebbert, M., Miami University, Oxford, OH

Ebbole, D., Texas A\&M University, College Station, TX

Eisenback, J., Virginia Polytechnic Institute, Blackburg, VA

Ellingboe, A. H., University of Wisconsin, Madison, WI

Elliot, M., L., University of Florida, Ft. Lauderdale, FL

Ennos, R., University of Edinburgh, Scotland

Essenberg, M., Oklahoma State University, Stillwater, OK

Fang, E., Clemson University, Clemson, SC

Feldstein, P., University of California, Davis, CA

Ferrandino, F. J., Connecticut Agricultural Experiment Station, New Haven, CT

Fox, S., Agriculture and Agri-Food Canada, Swift Current, SK, Canada

Francl, L., North Dakota State University, Fargo, ND

Fraedrich, S., USDA Forest Service, Athens, GA

Fulbright, D., Michigan State University, East Lansing, MI

Gabriel, D., University of Florida, Gainesville, FL

Gadoury, D., Cornell University, Geneve, NY

Gaunt, R. E., Lincoln University, New Zealand

Gitaitis, R., Coastal Plains Experimental Station, Tifton, GA

Gilbert, J., Agriculture and Agri-Food Canada, Winnipeg, MB, CA

Giovannoni, J., Texas A\&M University, College Station, TX

Gold, S., University of Georgia, Athens, GA

Gonsalves, D., Cornell University, Geneva, NY

Gonzalez, C. F., Texas A\&M University, College Station, TX

Goodman, R., University of Wisconsin, Madison, WI

Gordon, T. R., University of California, Davis, CA

Gottwald, T., USDA, ARS, Orlando, FL

Graham, T., Ohio State University, Columbus, $\mathrm{OH}$

Grau, C., University of Wisconsin, Madison, WI

Gray, S., Cornell University, Ithaca, NY

Groth, J. V., University of Minnesota, St. Paul, MN

Grove, G., Washington State University, Wenatchee, WA

Grybauskas, A., University of Maryland, College Park, MD

Haber, S., Agriculture and Agri-Food Canada, Winnipeg, MB, Canada

Hadidi, A., USDA, ARS, National Germplasm Lab., Beltsville, MD

Hammerschmidt, R., Michigan State University, East Lansing, MI

Hammond, J., USDA, ARS, Florist and Nursery Crops, Beltsville, MD

4 PHYTOPATHOLOGY 
Hammond, R., USDA, ARS, Molecular Plant Pathology Lab, Beltsville, MD

Hansen, E., Oregon State University, Corvallis, OR

Harder, D. E., Agriculture and Agri-Food Canada, Winnipeg, MB, Canada

Harkins, K., Iowa State University, Ames, IA

Harman, G., Cornell University, Geneva, NY

Harrington, T. C., Iowa State University, Ames, IA

Hartung, J., USDA, ARS, Beltsville, MD

Hausbeck, M., Michigan State University, East Lansing, MI

He, S. Y., Michigan State University, East Lansing, MI

Heath, M., University of Toronto, ON, Canada

Hickey, K., Penn State University, Biglerville, PA

Hiebert, E., University of Florida, Gainesville, FL

Higgins, V., University of Toronto, Toronto, ON, Canada

Hirano, S., University of Wisconsin, Madison, WI

Hobson, K., University of Wisconsin, Madison, WI

Hoch, H., NY State Agricultural Experiment Station, Geneva, NY

Hsu, H., USDA, ARS, US National Arboretum, Beltsville, MD

Huber, D., Purdue University, West Lafayette, IN

Huettel, R., USDA APHIS PPQ, Oxford, NC

Hughes, G., University of Edinburgh, Scotland

Hunt, A., University of Kentucky, Lexington, KY

Hutcheson, S., University of Maryland, College Park, MD

Illman, B, USDA Forest Products Lab, Madison, WI

Isakeit, T., Texas A\&M University, Weslaco, TX

Ishiguro, K., National Agric. Research Center, Tsukuba, Japan

Ishimaru, C., Colorado State University, Fort Collins, CO

Jabaji-Hare, S, Macdonald Campus of McGill University, Ste.

Anne Bellevue, QC, Canada

Jaffee, B., University of California, Davis, CA

Janisiewicz, W. J., USDA-ARS, Kearneysville, WV

Jarosz, A. M. , Michigan State University, East Lansing, MI

Jarvis, W., Agriculture and Agri-Food Canada, Harrow, ON, Canada

Jasalavich, C., 88 Broadway St., Arlington, MA

Jeffers, S., Clemson University, Clemson, SC

Jenkinson, P., Harper Adams Agricultrual College, Stropshire, United Kingdom

Johnson, D., Washington State University, Pullman, WA

Jones, A., Michigan State University, East Lansing, MI

Jones, J., University of Florida, Bradenton, FL

Jordan, R., USDA, ARS, Beltsville, MD

Judelson, H., University of California, Riverside, CA

Keen, N., University of California, Riverside, CA

Keller, N., Texas A\&M University, College Station, TX

Kenerly, C., Texas A\&M University, College Station, TX

Kimbrough, J., University of Florida, Gainesville, FL

Kobayashi, D., Rutgers University, New Brunswick, NJ

Kohn, L., University of Toronto, Mississauga, ON, Canada

Kronstad, J., University British Columbia, Vancouver, BC, Canada

Krupinsky, J., Northern Great Plains Research Station, Mandan, ND

Lacy, G., VPI \& SU, Blacksburg, VA

Lamari, L., Agriculture and Agri-Food Canada, Winnipeg, MB, Canada

Larkin, R., USDA, ARS, Beltsville, MD

Laurence, J., Cornell University, Ithaca, NY

Lee, I. M., USDA, ARS, PSI-BARC-West, Beltsville, MD

Legard, D., University of Florida, Dover, FL

Leonard, K., University of Minnesota, St. Paul, MN

Leslie, J., Kansas State University, Manhattan, KS

Leung, H., Washington State University, Pullman, WA

Levy, M., Purdue University, West Lafayette, IN

Lindbo, J., Pacific Lutheran University, Tacoma, WA

Lindow, S., University of California, Berkeley, CA
Liu, J. Q., Agriculture and Agri-Food Canada, Winnipeg, MB, Canada

Livingston, W., University of Maine, Orono, ME

Loesch-Fries, S. , Purdue University, West Lafayette, IN

Louws, F., North Carolina State University, Raleigh, NC

Manocha, M., Brock University, St. Catherines, ON, Canada

Marchetti, M., USDA, ARS, Rice Research, Beaumont, TX

Marois, J., University of Florida, Quincy, FL

Marshall, D., Texas A\&M University, Dallas, TX

Martelli, G., Universita di Patologia Vegetale, Bari, Italy

Martin, R., USDA, ARS, Corvallis, Corvallis, OR

Martyn, R., Texas A\&M University, College Station

Matheron, M., University of Arizona, Yuma, AZ

Maxwell, D. L., University of Wisconsin, Madison, WI

Mazzola, M., USDA, ARS, Wenatchee, WA

McCallum, B., University of Manitoba, Winnipeg, MB, Canada

McClure, M., University of Arizona, Tucson, AZ

McGawley, E., Louisiana State University, Baton Rouge, LA

McGrath, M. T., Cornell University, Riverhead, NY

McIntosh, R. A., University of Sydney, Camden, Australia

McManus, P., University of Wisconsin, Madison, WI

McNabb, Jr., H. S., Iowa State University, Ames, IA

Mendgen, K., Universitat Konstanz, Germany

Menzies, J., Agriculture and Agri-Food Canada, Winnipeg, MB, Canada

Mihail, J., University of Missouri, Columbia, MO

Milgroom, M., Cornell University, Ithaca, NY

Miller, D., Agriculture \& Agri-Food Canada, Ottawa, ON, Canada

Miller, S., Ohio State University, Wooster, $\mathrm{OH}$

Mims, C., The University of Georgia, Athens, GA

Mitchell, D. J., University of Florida, Gainesville, FL

Moore, L., Oregon State University, Corvallis, OR

Mora-Aguilera, G., Instituteo de Fitosanidad, Montecille, Mexico

Moyer, J., North Carolina State University, Raleigh, NC

Mundt, C., Oregon State University, Corvallis, OR

Munkvold, G. P., Iowa State University, Ames, IA

Murphy, J. F., Auburn University, Auburn, AL

Murray, T., Washington State University, Pullman, WA

Nelson, E., Cornell University, Ithaca, NY

Nelson, S. C., University of Hawaii, Honolulu

Nemec, S., USDA, ARS, Ft. Pierce, FL

Northover, J., Agriculture and Agri-Food Canada,Vineland

Station, ON, Canada

Nutter, F., Iowa State University, Ames, IA

O’Donnell, K., USDA, ARS, NCAUR, Peoria, IL

Ocamb, C., University of Minnesota, St. Paul, MN

Ostergaard, H., Riso National Laboratory, Denmark

Otrysko, B. Serv. des Sci Tech. de la Pomme de Terre, Les

Buissons, QC, Canada

Oudemans, P., Rutgers University, Chatsworth, NJ

Owens, R. A., USDA, ARS, Beltsville, MD

Panaccione, D., West Virginia University, Morgantown, WV

Parke, J. L., University of Wisconsin, Madison, WI

Parry, D., Harper Adams Agricultrual College, England

Pataky, J., University of Illinois, Urbana, IL

Payne, G., North Carolina State University, Raleigh, NC

Peever, T., Cornell University, Ithaca, NY

Pennington, R., USDA, ARS, Temple, TX

Pennypacker, B., Penn State University, University Park, PA

Perkins, J., DeKalb Genetics, DeKalb, IL

Pfender, W., Kansas State University, Manhattan, KS

Pierce, M., Oklahoma State University, Stillwater, OK

Pierson, L. S., University of Arizona, Tucson. AZ

Pinkerton, J., USDA, ARS, Corvallis, OR

Pirone, T., University of Kentucky, Lexington, KY

Powell, G., Imperial College at Silwood Park, England

Powelson, M., Oregon State University, Corvallis, OR 
Powers, T. O., University of Nebraska, Lincoln, NE

Pruvost, O., CIRAD FLHOR, Laboratoire de Phytopathologie, Reunion Island, France

Punja, Z. K., Simon Fraser University, Burnaby, BC, Canada

Purcell, A. H., University of California, Berkeley, CA

Quigley, N., University of Tennessee, Knoxville, TN

Reeleder, R., Agriculture and Agri-Food Canada, Delhi, ON, Canada

Roberts, D., Bioscience Bldg, Beltsville, MD

Rochon, D. M., Agriculture and Agri-Food Canada, Vancouver, BC, Canada

Roossinck, M. J., Noble Foundation, Ardmore, OK

Rosskopf, E., University of Florida, Gainesville, FL

Rouse, D., University of Wisconsin, Madison, WI

Rowhani, A., University of California, Davis, CA

Rupe, J., University of Arkansas, Fayetteville, FL

Samac, D. A., University of Minnesota, St. Paul, MN

Sanfacon, H., Agriculture and Agri-Food Canada, Vancouver, BC, Canada

Savary, S., International Rice Research Institute, Manila, Philippines

Schell, M., University of Georgia, Athens, GA

Scherm, H., Iowa State University, Ames, IA

Schisler, D., USDA, ARS, Peoria, IL

Schmitthenner, A. F., Ohio State University, Wooster, OH

Scott-Craig, J., Michigan State University, East Lansing, MI

Sequin-Swartz, G., Agriculture and Agri-Food Canada, Saskatoon, SK, Canada

Schaad, N., USDA, ARS, Frederick, MD

Schisler, D., USDA, ARS, NCAUR, Peoria, IL

Shamoun, S., Pacific Forestry Centre, Victoria, BC, Canada

Shaner, G., Purdue University, West Lafayette, IN

Shaw, M. W., University of Reading, Reading, England

Sherwood, J. L., Oklahoma State University, Stillwater, OK

Shtienberg, D., The Volcani Center, Bet-Dagan, Israel

Singh, R., Agriculture and Agri-Food Canada, Fredericton, MB, Canada

Skinner, D. Z., Kansas State University, Manhattan, KS

Smilanick, J., USDA, ARS Horticulutal Crops Research, Fresno, CA

Smith, B., USDA,ARS, Small Fruit Research Station, Poplarville, MS

Smith, D., University of Kentucky, Lexington, KY

Spatafora, J. W., Oregon State University, Corvallis, OR

Spotts, R., Mid-Columbia AREC, Hood River, OR

Stall, R., University of Florida, Gainesville, FL
Stanosz, G., University of Wisconsin, Madison, WI

Steffenson, B., North Dakota State University, Fargo, ND

Stenger, D., Northern Illinois University, Dekalb, IL

Stockwell, V., USDA, ARS, Horticultural Crops Research Lab, Corvallis, OR

Sumner, D., Coastal Plains Exp. Station, Tifton, GA

Sutton, T., North Carolina State University, Raleigh, NC

Swart, W., The University of The Orange Free State, Republic of South Africa

Szabo, L., University of Minnesota, St. Paul, MN

Taylor, D., University of Virginia, Charlottesville, SC,

TeBeest, D., University of Arkansas, Fayetteville, AR

Tekauz, A., Agriculture and Agri-Food Canada, Winnipeg, MB, Canada

Thomson, S., Utah State University, Logan, UT

Timper, P., University of Arkansas, Fayetteville, AR

Tolin, S., VPI \& State University, Blacksburg, VA

Trudgill, D., Scottish Crop Research Institute, Dundee, Scotland

Turgeon, B. G., Cornell University, Ithaca, NY

Tuttle McGrath, M., Cornell University, Riverhead, NY

Tuzun, S., Auburn University, Auburn, AL

Uknes, S., Ciba-Geigy Corporation, Research Triangle Park, NC

Ullman, D. E., University of California, Davis, CA

Upchurch, R. G., North Carolina State University, Raleigh, NC

Van Alfen, N, Texas A\&M University, College Station, TX

Van Bruggen, A., University of California, Davis, CA

VanEtten, H., University of Arizona, Tucson, AZ

Vincelli, P., University of Kentucky, Lexington, KY

Walton, J., Michigan State University, East Lansing. MI

Weidemann, G., University of Arkansas, Fayetteville, AR

White, D., University of Illinois, Urbana, IL

Wilcox, W., Cornell University, Geneva, NY

Williams, W. P., USDA-ARS, CHPRU, Mississippi State, MS

Wilson, J., USDA, ARS, Coastal Plain Experimental Station, Tifton, GA

Wilson, M., Auburn University, Auburn, AL

Windels, C., University of Minnesota, Crookston, MN

Wingfield, M., Moni Professor of Forest Pathology, Bloemfontein, South Africa

Wisler, G. , USDA-ARS, U.S. Agricultural Research Station, Salinas, CA

Woloshuk, C., Purdue University, West Lafayette, IN

Yang, X. B., Iowa State University, Ames, IA

Young, N., University of Minnesota, St. Paul, MN

Yuen, G., University of Nebraska, Lincoln, NE

Zentmeyer, G. A., University of California, Riverside, CA 